\title{
Lesões em áreas de furca: fatores etiológicos, diagnóstico e tratamento
}

Lesions in furcation areas: etiological factors, diagnosis and treatment Lesiones en áreas de furcación: factores etiológicos, diagnóstico y tratamiento

\section{Ariana Larissa de Moura RODRIGUES ${ }^{1}$ \\ Ana Carolina de Sá Gomes Cruz SOUZA ${ }^{1}$ Jéssica Gomes Alcoforado de MELO $^{2}$ Diego Moura SOARES}

${ }^{I}$ Discente do Curso de Odontologia da Faculdade de Integração do Sertão (FIS) 56909-205 Serra Talhada - PE, Brasil ${ }^{2}$ Professora do Curso de Odontologia da Faculdade de Integração do Sertão (FIS) 56909-205 Serra Talhada-PE, Brasil ${ }^{3}$ Professor Adjunto da Faculdade de Integração do Sertão (FIS) 56909-205 Serra Talhada - PE, Brasil

\section{Resumo}

As lesões de furca ocorrem quando a doença periodontal atinge a área de bifurcação dos dentes multirradiculares causando a destruição óssea e perda de inserção no espaço inter-radicular. Existem diversos fatores etiológicos que influenciam no aparecimento dessas lesões e até os dias de hoje o tratamento desse tipo de injúria ainda é um desafio na clínica odontológica. O objetivo deste artigo foi listar, através de uma revisão da literatura, os fatores que influenciam na etiologia da lesão de furca, bem como o seu diagnóstico, prognóstico e tratamento. Fatores como características morfológicas do dente e raiz e deficiência no controle do biofilme, que podem contribuir para o seu aparecimento. Além de diversos tipos de procedimentos e técnicas têm sido propostas para o tratamento das lesões de furca, seja mais ou menos conservadores.

Descritores: Defeitos da Furca; Diagnóstico; Doenças Periodontais.

\section{Abstract}

Furcation lesions occur when periodontal disease reaches the bifurcation area of multiradicular teeth causing bone destruction and loss of insertion in the inter-radicular space. There are several etiological factors that influence the appearance of these lesions and even today the treatment of this type of injury is still a challenge in the dental clinic. The purpose of this article was to list, through a literature review, the factors that influence the etiology of the furcation injury, as well as its diagnosis, prognosis and treatment. Factors such as tooth and root morphological characteristics and deficiency in biofilm control, which can contribute to its appearance. In addition to several types of procedures and techniques, they have been proposed for the treatment of furcation injuries, whether more or less conservative.

Descriptors: Furcation Defects; Diagnosis; Periodontal Diseases.

\section{Resumen}

Las lesiones de furca se producen cuando la enfermedad periodontal alcanza el área de bifurcación de los dientes multirradiculares causando destrucción ósea y pérdida de inserción en el espacio interradicular. Existen varios factores etiológicos que influyen en la apariencia de estas lesiones e incluso hoy en día el tratamiento de este tipo de lesión sigue siendo un desafío en la clínica dental. El propósito de este artículo fue enumerar, a través de una revisión de la literatura, los factores que influyen en la etiología de la lesión por furca, así como su diagnóstico, pronóstico y tratamiento. Factores como las características morfológicas de los dientes y las raíces y la deficiencia en el control de biopelículas, que pueden contribuir a su aparición. Además de varios tipos de procedimientos y técnicas, se han propuesto para el tratamiento de lesiones por furca, ya sean más o menos conservadoras.

Descriptores: Defectos de furcación; Diagnóstico; Enfermidades periodontales.

\section{INTRODUÇÃO}

A área de furca pode ser definida como uma região anatômica característica de dentes multirradiculares, onde as raízes começam a se dividir do tronco radicular ${ }^{1}$. Quando a doença periodontal atinge os tecidos de suporte e sustentação de dentes multirradiculares, causando a reabsorção/ destruição óssea adjacente e perda de inserção no espaço inter-radicular, provoca-se a chamada lesão de furca. Existem diversos fatores etiológicos e locais que auxiliam no envolvimento da região de furca, com consequente perda de inserção horizontal, com variados graus de destruição ${ }^{2}$.

O diagnóstico, avaliação e prognóstico correto são fundamentais para o estabelecimento do adequado tratamento periodontal das lesões de furca, afetando diretamente o curso e o sucesso da terapia ${ }^{3}$. A partir da avaliação clínica, juntamente com radiografias da lesão de furca, pode-se utilizar a sonda convencional, assim como de Nabers, para o acesso da região com a ponta ativa na extremidade horizontal da furca ${ }^{4}$.

Mesmo após a inspeção detalhada da lesão de furca, o tratamento ainda é desafiador ${ }^{2,5}$. Pretende-se, com a terapia periodontal, eliminar o biofilme das superfícies radiculares e restabelecer uma anatomia adequada da região afetada, para que exista uma correta higienização e controle do biofilme, sendo este o principal objetivo dos tratamentos das lesões de furca ${ }^{6}$. Existem diversos tipos de tratamentos para lesões na região de furca, podendo ser divididos em conservadores (raspagem e alisamento radicular), ressectivos (tunelização, hemissecção e ressecção radicular)e os regeneradores ${ }^{2}$.

Dessa forma, o objetivo deste artigo foi listar, através de uma revisão da literatura existente, os fatores que influenciam na etiologia da lesão de furca, bem como o seu diagnóstico, prognóstico e tratamento.

\section{MATERIAL E MÉTODO}

Este estudo reporta uma revisão de literatura que teve como objetivo relatar os fatores etiológicos e predisponentes das lesões de furca, bem como o seu diagnóstico, classificação e principais opções de tratamento correlacionando com o grau da lesão. As buscas foram realizadas sem restrição temporal nas bases eletrônicas de dados: Scopus, Lilacs, PubMed/MEDLINE e no Scielo. As publicações foram em língua portuguesa e inglesa, empregando as seguintes palavras-chave: "furcation defects", "furcation involvement", "class I furcation involvement", "class I furcation defects", "class II 
furcation involvement", "class II furcation defects", "class III furcation involvement", "class III furcation defects". Os critérios de inclusão foram artigos que abordaram os fatores etiológicos, diagnóstico, classificação ou tratamento das lesões de furca.

\section{RESULTADOS E DISCUSSÃO}

\section{- Fatores etiológicos e predisponentes}

Algumas considerações anatômicas e fatores predisponentes podem contribuir para o surgimento e/ou intensidade do processo de destruição periodontal na lesão de furca. Conhecer esses fatores propicia uma maior segurança no planejamento do tratamento e condução do caso, favorecendo o prognóstico do dente envolvido ${ }^{7}$. Os principais fatores etiológicos e predisponentes incluem acúmulo de biofilme, concavidades radiculares, projeção cervical do esmalte, pérolas de esmalte, altura do tronco radicular, condições endodônticas e trauma oclusal.

O biofilme bacteriano pode ser caracterizado por uma comunidade microbiana relativamente indefinida associada à superfície de qualquer material duro não descamativo (dente). O seu acúmulo é descrito como sendo o principal fator etiológico das doenças periodontais, incluindo a lesão de furca. A sua presença provoca uma inflamação crônica local, resultando na perda de inserção, atingindo a região interradicular e dificultando ainda mais a sua remoção e manutenção da higiene bucal pelo paciente e o controle por parte do profissional ${ }^{4}$.

As concavidades radiculares favorecem $o$ acúmulo de biofilme, além de dificultar a remoção mecânica durante a terapia de raspagem e a higiene bucal por parte do paciente. A prevalência de concavidades radiculares é bastante comum nas raízes mesiovestibulares, nos molares superiores, e nas raízes mesiais dos molares inferiores. Essas regiões anatômicas possuem maior quantidade de cemento do que as convexidades constituindo de nichos de retenção de biofilme ${ }^{8}$.

Como o nome já diz, são projeções de esmalte na região cervical do dentes. Essas projeções podem facilitar a invasão microbiana, predispondo a colonização da furca, visto que as fibras do ligamento periodontal não se inserem no esmalte .

As pérolas de esmalte podem estar presentes tanto no espaço interradicular, quanto em qualquer face do dente e são definidas como projeções globulares de esmalte ${ }^{7}$. Da mesma forma que nas projeções cervicais de esmalte, quando as pérolas de esmalte localizam-se nas proximidades da furca, não existe sobre elas inserção conjuntiva e, sim, aderência epitelial por meio de hemidesmossomos, fazendo com que se tornem fatores predisponentes à invasão de furca ${ }^{10}$.

O tronco radicular é uma estrutura anatômica

que tem como limite superior a junção

esmalte-cemento e inferior o início da bifurcação radicular propriamente dita. Esta estrutura é comum a todos os dentes multirradiculares. Dentes com tronco radicular curto são mais vulneráveis à exposição da furca quando comparado aos dentes com tronco radicular longo. Entretanto, após exposição, o tratamento é mais complicado em dentes com tronco radicular longo, devido ao acesso dificultado à região .

Devido à presença de canais acessórios no teto da furca, lesões de furca de origem endodôntica devem ser consideradas. Se a polpa estiver infectada, lesões endoperiodontais podem ocorrer, levando ao aparecimento de lesão de furca. Nesses casos, o dente em questão não apresentará vitalidade pulpar e a lesão de furca normalmente é bem localizada, não atingindo o osso proximal ${ }^{10}$.

Os traumas oclusais são conseqüência de forças excessivas produzidas pelos músculos da mastigação. Os mesmos podem predispor a lesões de furca, sendo o aumento da mobilidade dentária, imagens radiolúcidas na região interradicular, atrelado à sondagem normal da região de furca, sinais típicos de trauma oclusal em molares ${ }^{7}$.

- Classificação, diagnóstico e prognóstico

As lesões de furca são comumente classificadas tendo como base a extensão da destruição horizontal dos tecidos periodontais. A classificação proposta por Hamp et al. ${ }^{11}$ até hoje é a mais utilizada; nela, os autores classificaram as lesões em: Classe I, quanto a perda horizontal do tecido de suporte é menor que $3 \mathrm{~mm}$; Classe II, caracterizada pela perda horizontal maior ou igual a 3 $\mathrm{mm}$; e Classe III, quando existe uma perda horizontal dos tecidos periodontais que atravessa completamente a furca.

Outra classificação foi proposta por Ramfjord e $\mathrm{Ash}^{12}$, baseada na de Hamp et al. ${ }^{11}$. Os autores classificaram as lesões de furca em: Classe I - lesão inicial em que a perda de inserção na região da furca é menor que $2 \mathrm{~mm}$, ou seja, menor que $1 / 3$ do tamanho do dente; Classe II - lesão maior que $2 \mathrm{~mm}$, ou seja, maior que $1 / 3$ do dente, mas ainda não atravessa a furca; Classe III - envolvimento de toda a extensão da furca.

Com exceção da classificação proposta por Tarnow e Fletcher ${ }^{13}$, todas as outras avaliam apenas a destruição dos tecidos periodontais de forma horizontal. A partir da necessidade de uma análise vertical do defeito, os autores acima mencionados classificaram as lesões de furca em Classe A, B e C, onde, na classe $\mathrm{A}$, a distância do teto da furca ao fundo do defeito é de 0 a $3 \mathrm{~mm}$, classe B é de 4 a 7 $\mathrm{mm}$, e, na classe $C$, essa distância é de $7 \mathrm{~mm}$ ou mais.

Assim, o diagnóstico e o prognóstico para o tratamento das lesões de furca estão relacionados com a profundidade de sondagem da área comprometida e do grau de envolvimento da mesma. 
O diagnóstico adequado deve ser realizado, com um exame clínico detalhado e análise radiográfica e a quantidade de destruição periodontal mensurada com o auxílio de uma sonda milimetrada do tipo Nabers favorecendo a classificação do grau de envolvimento da área avaliada ${ }^{4}$.

O exame radiográfico da região de furca permite avaliar a quantidade de osso presente, altura da crista óssea alveolar, integridade da lâmina dura. Todos estes parâmetros auxiliam no correto diagnóstico e confirma os achados encontrados a partir da avaliação clínica ${ }^{2}$.

O prognóstico do tratamento de furca está diretamente relacionado com o grau da severidade do envolvimento da mesma. Assim, quanto maior o grau de classificação da lesão, menor a taxa de sucesso do tratamento periodontal conservador e consequentemente do prognóstico a longo prazo do dente afetado ${ }^{14}$.

\section{- Tratamento das lesões de furca}

$\mathrm{O}$ tratamento das lesões de furca ainda é uma tarefa bastante difícil e conflitante na terapia periodontal. É sabido que o mesmo deve ter como objetivos principais a eliminação do biofilme das superfícies radiculares expostas, proporcionar um melhor controle do biofilme por parte do paciente e, sempre que possível, restabelecer estruturas anatômicas perdidas com a evolução da doença. Dessa forma, a escolha do tratamento depende de alguns fatores, como anatomia radicular, anomalias, grau de comprometimento dentário e principalmente o grau de envolvimento de furca, assim como da expectativa e anseios do paciente frente ao tratamento indicado ${ }^{15}$.

São propostos na literatura diversos tipos de tratamentos para as lesões de furca, desde os conservadores que envolvem a raspagem $\mathrm{e}$ alisamento radicular, com ou sem cirurgia, odontoplastia e osteoplastia; os ressectivos que envolvem a tunelização, hemissecção radicular e ressecção radicular e os regenerativos que envolvem a Regeneração Tecidual Guiada (RTG) e enxerto ósseo $^{2}$. Os objetivos do tratamento para esses defeitos são para facilitar a manutenção, prevenir a perda adicional de inserção e fechar os defeitos de furca, mas é preciso deixar claro que o controle da infecção é essencial antes de qualquer procedimento ressectivo ou regenerativo'.

\section{- Raspagem e alisamento radicular (RAR)}

Trata-se de um tipo de tratamento conservador que tem como objetivo principal a descontaminação do cemento e consequentemente o restabelecimento da saúde periodontal na região de furca. Este tratamento está indicado para todas as classes de lesão de furca, porém, apresenta um melhor prognóstico quando realizado em lesões de furca classe I. Isso se deve à profundidade rasa das bolsas, atrelado a um pequeno grau de destruição óssea, facilitando, assim, a remoção do agente infeccioso e propiciando a reparação ${ }^{10}$. Para os tratamentos de lesão de furca grau I, percebeu-se o sucesso no uso de raspagem e alisamento radicular com ou sem o auxílio dos procedimentos de osteoplastia e odontoplastia. Além disso, descrevem uma taxa de sobrevida de $100 \%$ no período analisado para o estudo (5 anos) quando se observa o uso de RAR com esse método não cirúrgico ${ }^{11}$.

O sucesso e redução e/ou eliminação das áreas retentivas dependem concomitantemente da utilização de higiene bucal dos pacientes ${ }^{11}$. Algumas vezes esses métodos podem não ser suficientes para eliminar as bolsas periodontais ou não haver a correta higienização, lançando-se mão do debridamento cirúrgico simples, procedimento conservador que ocorrerá o rebatimento do retalho, possibilitando a visualização da zona de furca ${ }^{16}$.

\section{- Plastia da furca}

Este tipo de tratamento consiste em reduzir o componente ósseo e dentário da entrada da furca, horizontalmente, facilitando a higienização dos pacientes a partir de uma melhoria na anatomia radicular da região de furca. A plastia só será feito em molar inferior ${ }^{15}$. É utilizada principalmente nas furcas vestibulares e linguais. Nas superfícies proximais, o acesso é limitado para esse tipo de tratamento10. Esse tratamento é indicado para obter a redução do acúmulo de placa bacteriana ${ }^{17}$.

A indicação da realização da plastia em área de furca fica restrita a lesões incipientes grau I ou II. $\mathrm{O}$ procedimento envolve um acesso da área interradicular através de retalho, além de raspagem e alisamento radicular9. A plastia de furca também pode ser usada com a odontoplastia (remoção de materiais dentários) e osteoplastia (recontorno da crista óssea alveolar) ${ }^{17}$.

\section{- Tunelização}

Tem como principal objetivo a obtenção de uma furca totalmente aberta para facilitar a higienização da área. É indicada para molares inferiores que possuem um tronco radicular curto, um amplo ângulo de separação e uma grande divergência entre as raízes mesial e distal, em lesões de furca grau $\mathrm{II}^{10}$. No entanto, esse procedimento apresenta um elevado risco de sensibilidade radicular e desenvolvimento de cáries no interior dos túneis preparados ${ }^{11}$.

Esta técnica não requer tratamento endodôntico e ou reconstruções protéticas prévias, porém, apresenta como desvantagem a exposição dos canais laterais e ou cáries radiculares, exigindo tratamento endodôntico posteriormente ${ }^{4}$. Outra complicação comum é a fratura radicular. Taxas de sobrevivência dos dentes tratados com tunelização ao longo de cinco anos são entre $57 \%$ a $92 \%$ e a maioria dos dentes são perdidos devido a lesões cariosas ${ }^{18}$.

Estudos têm demonstrado resultados 
satisfatórios do uso desta técnica no tratamento de lesões de furca grau III. As restrições anatômicas favorecem o uso desta técnica nos primeiros molares inferiores, apesar de também poder ser empregada em molares superiores e segundos molares inferiores ${ }^{19}$.

\section{- Ressecção radicular}

Consiste na remoção de uma das raízes de um dente multirradicular, eliminando a furca envolvida na lesão. Esta técnica é mais indicada para lesões mais avançadas de grau II ou III de furca e pode ser empregada tanto em molares superiores, quanto em inferiores ${ }^{14}$.

\section{- Hemissecção radicular}

Indicado para os casos de lesão de furca grau II e III, consiste na separação de raízes de dentes multirradiculares, podendo ser seguida da remoção de uma ou mais raízes, juntamente com suas porções coronárias do respectivo elemento dentário. Tanto a técnica de ressecção radicular, quanto a hemissecção, requer tratamento endodôntico prévio ao procedimento cirúrgico ${ }^{9}$.

Esse tipo de tratamento ainda se mostra em contraditória relacionado ao seu sucesso clínico no tratamento de lesões de furca grau III. Devido ser necessário de um procedimento multidisciplinar podendo comprometer o sucesso terapêutico ${ }^{19}$. O comprimento do tronco radicular, o comprimento, a divergência das raízes, e a quantidade de suporte ósseo remanescente ao redor de cada raiz devem ser considerados $^{20}$.

As falhas que mais ocorrem nos casos de ressecção e hemissecção radicular é a fratura radicular, seguida de cáries. As taxas de sucesso a longo prazo varia de 62 a $100 \%{ }^{18}$.

\section{- Regeneração Tecidual Guiada (RTG)}

Consiste na colocação de uma barreira física, com a finalidade de evitar que as células epiteliais povoem primeiro a superfície do defeito após a realização de um debridamento da área. Este fato se baseia no princípio que considera que o tipo de cicatrização do defeito periodontal é determinado pelo primeiro tipo de célula que repovoa a superfície radicular. Dessa forma, a RTG visa eliminar o contato das células dos tecidos epitelial e conjuntivo gengival com a superfície radicular nos estágios iniciais de cicatrização, a partir do uso de membranas que atuam como barreira mecânica, pois estas não têm capacidade de regenerar os tecidos perdidos ${ }^{10}$.

As membranas utilizadas na RTG podem ser reabsorvíveis e não reabsorvíveis. E o sucesso da técnica depende de características individuais do paciente, como controle da inflamação, padrão de higiene $^{21}$ e potencial de cicatrização do paciente, além de fatores relacionados à técnica e ao defeito (profundidade de sondagem, morfologia do defeito, anatomia radicular, tipo de membrana utilizada) ${ }^{14}$.

RTG como tratamento de lesões de furca grau II maxilares e grau III maxilar e mandibular é imprevisível $^{10}$, no entanto, resultados clínicos satisfatórios são esperados no tratamento de lesão grau II mandibular, seguido dos molares maxilares, mas com efeitos clínico limitado, devido os defeitos interproximais pela dificuldade do acesso e da adaptação do material ${ }^{22}$.

\section{- Enxerto ósseo}

É uma modalidade regenerativa que apresenta a vantagem de poder reconstruir a anatomia perdida, além de um bom prognóstico. Consiste no deslocamento total do retalho, remoção do tecido de granulação, debridamento das superfícies radiculares e o preenchimento do defeito com enxerto ósseo ${ }^{4}$.

- Retalho Reposicionado Coronalmente (RRC)

É uma técnica cirúrgica descrita a vários anos com a indicação de melhorar procedimentos periodontais estéticos. Esta técnica consiste no deslocamento coronalmente do retalho com posterior sutura para fixação do mesmo em nova posição até a cicatrização. O RRC pode ser utilizado associado ou não a enxerto de tecido conjuntivo e biomateriais. A técnica clássica do RRC preconizava a realização de incisões relaxantes na mesial e distal, sendo assim o sucesso do procedimento depende em muito do suprimento sanguíneo. Nesta técnica o suprimento é proveniente da base do retalho, que deverá ser confeccionado com uma forma trapezoidal de base larga, já que o tecido é colocado sobre a superfície radicular não vascularizada ${ }^{23}$.

A utilização de qualquer uma das técnicas regenerativas, para o tratamento de lesões de furca grau III, ainda possui evidências científicas pouco limitadas e os estudos mostram resultados poucos favoráveis e restrita previsibilidade, tornando esse método imprevisível ${ }^{24,25}$.

\section{- Exodontia}

É indicada quando o dente afetado não irá melhorar o qualquer plano de tratamento ${ }^{11}$. Quando não permita ao paciente uma adequada manutenção de higienização, para indivíduos que não mantém um adequado controle de placa, tem um índice de atividade de cárie alto, que não tenham condições financeiras para arcar com terapias mais complexas ${ }^{9}$.

A escolha do melhor tratamento para cada tipo de lesão tem que ser levado em conta os aspectos individuais de cada paciente. Sendo importante verificar diversas questões, como a habilidade de manter o controle da placa bacteriana, o risco de desenvolvimento de cárie, as características morfológicas da raiz ${ }^{26,27}$.

\section{CONCLUSÃO}

É possível concluir que as lesões de furca são lesões que podem aparecer em decorrência da periodontite, levando a uma maior probabilidade da perda dentária. E que diversos fatores, como características morfológicas do dente/raiz e 
deficiência no controle do biofilme, que podem contribuir para o seu aparecimento.

Além disso, diversos tipos de procedimentos e técnicas têm sido propostas para o tratamento das lesões de furca, seja mais ou menos conservadores. A opção da técnica de tratamento pelo clínico deve sempre está baseada no seu diagnóstico e classificação das lesões, bem como a capacidade do paciente em manter a região livre do acúmulo do biofilme.

\section{REFERÊNCIAS}

1. Deliberador TM, Nagata MJH, Furlaneto FAC, Messora MR, Bosco AF, Garcia VG et al. Abordagem conservadora no tratamento dos defeitos de furca. RSBO. 2008;5(8):49-55.

2. Silva GP, Sousa Neto AC, Pereira AFV, Alves CMC, Pereira ALA, Serra LLL. Classificação e tratamento das lesões de furca. Rev Ciênc Saúde. 2014;16(2):112-28.

3. Nibali L, Zavattini A, Nagata K, Di Iorio A, Lin $\mathrm{GH}$, Needleman I, et al. Tooth loss in molars with and without furcation involvement - a systematic review and meta-analysis. J Clin Periodontol. 2016;43(2):156-66.

4. Artacho MCI, Arambulo GM. Defectos de furcación. Etiología, diagnóstico y tratamiento. Rev Estomatol Herediana. 2010;20(3):172-78.

5. Pereira SG, Pinho MM, Almeida RF. Regeneração periodontal em lesões de furcarevisão da literatura. Rev port estomatol med dent cir maxilofac. 2012;53(2):123-32.

6. Queiroz LA, Casarian RCV, Daddoub SM, Tatakis DN, Enilson AS, Kumar PS. Furcation Therapy with Enamel Matrix Derivative: Effects on the Subgingival Microbiome. J Periodontol. 2017;88(7):617-25.

7. Vieira TR, Costa FO, Zenóbio EG, Soares RV. Anatomia radicular e suas implicações na terapêutica periodontal. Rev Periodontia 2009; 19(1):7-13.

8. Bower RC. Furcation morphology relative to periodontal treatment. Furcation root surface anatomy. J Periodontol. 1979;50(7):366-74.

9. Newman M, Takei H, Klokkevold P, Carranza F. Periodontia clínica. 12. ed. São Paulo: Elservier; 2016.

10. Lindhe J, Karring T, Lang NP. Tratado de periodontia clínica e implantodontia oral. 5. ed; Rio de Janeiro: Guanabara Koogan;2010.

11. Hamp SE, Nyman S, Lindhe J. Periodontal treatment of multirroted teeth. Result after 5 years. J Clin Periodontol. 1975;2(3):126-35.

12. Ramjford SP, Ash MM. Periodontology and Periodontics. Philadelphia: W.B. Saunders Co; 1979.

13. Tarnow D, Fletcher P. Classification of the vertical component of furcation involvement. $\mathrm{J}$
Periodontol. 1984;55(5):283-84.

14. Walter C, Weiger R, Zitman NU. Periodontal surgery in furcation-involved maxillary molars revisited: an introduction of guidelines for comprehensive treatment. Clin Oral Investig. 2011;15(1):9-20.

15. Sallum AW, Cicareli AJ, Querido MRM, BastosNeto FVR. Periodontia e implantodontia Soluções estéticas e recursos clínicos. Rio de Janeiro: Napoleão; 2010.

16. Graziani F, Gennai S, Karapetsa D, Rosini S, Filice N, Gabriele $\mathrm{M}$ et al. Clinical performance of access flap in the treatment of class II furcation defects. A systematic review and meta-analysis of randomized clinical trials. J Clin Periodontol. 2015;42(2):169-81.

17. Svärdström G, Wennström JL. Periodontal treatment decisions for molars: an analysis of influencing factors and long-term outcome. J Periodontol. 2000;71(4):579-85.

18. Huynh-Ba G, Kuonen P, Hofer D, Schmid J, Lang NP, Salvi GE. The effect of periodontal therapy on the survival rate and incidence of complications of multirooted teeth with furcation involvement after an observation period of at least 5 years: a systematic review. J Clin Periodontol. 2009;36(2):164-76.

19. Shirakata Y, Miron RJ, Nakamura T, Sena K, Shinohara $\mathrm{Y}$, Horai $\mathrm{N}$, et al. Effects of EMD liquid (Osteogain) on periodontal healing in class III furcation defects in monkeys. J Clin Periodontol. 2017;44(3):298-307.

20. Meyle J, Gonzales JR, Bödeker RH, Hoffmann T, Richter S, Heinz B et al. A randomized clinical trial comparing enamel matrix derivative and membrane treatment of buccal class II furcation involvement in mandibular molars. Part II: secondary outcomes. J Periodontol. 2004; 75(9):1188-95.

21. Jenabian N, Haghanifar S, Ehsani H, Zahedi E, Haghpanah M. Guided tissue regeneration and platelet rich growth factor for the treatment of Grade II furcation defects: A randomized doubleblinded clinical trial - A pilot study. Dent Res J (Isfahan). 2017;14(6):363-69.

22. Kinaia M, Steiger J, Neely AL, Shah M, Bhola M. Treatment of class II molar furcation involvement: meta-analyses of re-entry results. J Periodontol. 2011;82(1):413-28.

23. Correa A, Ferreira PS, Barboza R, Ribeiro EDP, Bittencourt S. Fatores que influenciam no sucesso da técnica do retalho posicionado coronalmente. Rev Bahiana Odonto; 2013;4(2):117-28.

24. Jepsen S, Gennai S, Hirschfeld J, Kalemaj Z, Buti J, Graziani F. Regenerative surgical treatment of furcation defects: A systematic review and Bayesian network meta-analysis of randomized clinical trials. J Clin Periodontol. 2020;47(Suppl 22):352-74. 
25. Reddy MS, Aichelmann-Reidy ME, Avila-Ortiz G, Klokkevold PR, Murphy KG, Rosen PS, et al. Periodontal regeneration - furcation defects: a consensus report from the AAP Regeneration Workshop. J Periodontol. 2015;86(2 Suppl):S131-3.

26. Casarin RCV, Ribeiro EDP, Nociti-Jr FH, Sallum AW, Ambrosano GMB, Sallum EA, et al. Enamel matrix derivative proteins for the treatment of proximal class II furcation involvements: a prospective 24-month randomized clinical trial. J Clin Periodontol; 2010;37(12):1100-109.

27. Hoffmann T, Richter S, Meyle J, Gonzales JR, Heinz B, Arjomand M et al. A randomized clinical multicentre trial comparing enamel matrix derivative and membrane treatment of buccal class II furcation involvement in mandibular molars. Part III: patient factors and treatment outcome. J Clin Periodontol. 2006;33(8):575-83.

\section{CONFLITO DE INTERESSES}

Os autores declaram não haver conflitos de interesse.

\section{AUTOR PARA CORRESPONDÊNCIA}

Diego Moura Soares

Rua Emiliano Braga, 635, Iputinga, 50670-380 Recife-PE, Brasil diegomsoares@hotmail.com
Submetido em 15/07/2020

Aceito em 16/12/2020 